ISSN 1112-9867

Available online at

http://www.jfas.info

\title{
BRILLOUIN INSTABILITY IN FIBER LASERS DOPED BY POWER
}

\author{
D. M. Bouras", A. Kellou, H. Leblond and F. Sanchez \\ Laboratoire d'Electronique Quantique, Faculté de Physique, Université des Sciences et \\ de la Technologie Houari Boumediene,B.P. 32 El-Alia Bab-Ezzouar 16111, Alger, \\ Algeria \\ Laboratoire POMA FRE CNRS 2988, Université d'Angers 2 Bd Lavoisier, 49000 \\ Angers, France \\ Université de Mhamed Bouggara de Boumerdes
}

Received: 25 November 2012 / Accepted: 02 December 2012 / Published online: 30 June 2012

\begin{abstract}
With the emergence of rare-earth doped fibers, and especially double-clad fibers, there is a renewed interest in Brillouin effect. First of all, the amplification of a continuous signal in a rare-earth doped fiber amplifier can generate high enough intensities to excite Brillouin effect and then to create a backscattered stokes wave. Such back-reflection is detrimental for amplifier applications and consequently it has been studied theoretically and experimentally. Recently, the low frequency self-pulsing instability resulting from Brillouin backscattering has been theoretically modelled [1]. Our main objective is to present a general model allowing to explain the origin of the dynamic instability arising in a fiber lasers as a consequence of Brillouin effect. The effect of Brillouin back scattering is theoretically analysed by two-coupled modes laser model. We consider the Fabry-Perot fiber laser cavity. The rich and complex dynamic behaviours are observed. In particular the quasi periodic dynamic is identified and studied.
\end{abstract}

Keywords: Stimulated Brillouin Scattering; high power fiber lasers, instabilities, quasi periodic.

Author Correspondence, e-mail: djouher.mallek@umbb.dz ICID: 1020708 


\section{LE MODELE DES AMPLITUDES COUPLEES (MAC).}

Dans ce travail, l'influence de l'effet Brillouin sur la dynamique d'un laser à fibre de puissance, dans une cavité type Fabry Pérot, est décrite par le modèle des amplitudes couplées. Cette approche consiste à considérer deux équations d'inversion de population afin de décrire l'évolution temporelle du milieu amplificateur. On ne tient pas compte des ordres supérieurs des composantes Stokes. Le gain de la diffusion Brillouin n'est plus considéré comme instantané [1]. Les ondes sont décrites en termes d'amplitudes des champs optiques et acoustique et des conditions aux limites. Dans sa forme finale, ce modèle d'amplitudes couplées (1) comprend huit équations, deux pour les inversions de population et six pour les amplitudes des champs optiques et acoustique:

$$
\begin{aligned}
& \frac{\partial d_{1}}{\partial t}=p-a_{1}\left(d_{1}+1\right)-2 d_{1}\left(\left|e_{p}^{ \pm}\right|^{2}+\left|e_{p}^{-}\right|^{2}+\beta\left(\left|e_{s}^{+}\right|^{2}+\left|e_{s}^{-}\right|^{2}\right)\right), \\
& \frac{\partial d_{2}}{\partial t}=\gamma p-a_{1}\left(d_{2}+1\right)-2 d_{2}\left(\left|e_{s}^{+}\right|^{2}+\left|e_{s}^{-}\right|^{2}+\beta\left(\left|e_{p}^{+}\right|^{2}+\left|e_{p}^{-}\right|^{2}\right)\right), \\
& \frac{\partial e_{a}^{ \pm}}{\partial t}=\alpha_{a}\left(e_{p}^{ \pm} e_{s}^{\mp *}-e_{a}^{ \pm}\right), \\
& \frac{\partial e_{p}^{ \pm}}{\partial t} \pm \frac{\partial e_{p}^{ \pm}}{\partial z}=-g_{c} e_{s}^{\mp} e_{a}^{ \pm}-\frac{\alpha_{c}}{2} e_{p}^{ \pm}+\frac{A_{c}}{2}\left(d_{1}+\beta d_{2}\right) e_{p}^{ \pm}, \\
& \frac{\partial e_{s}^{ \pm}}{\partial t} \pm \frac{\partial e_{s}^{ \pm}}{\partial z}=g_{c} e_{p}^{\mp} e_{a}^{\mp *}-\frac{\alpha_{c}}{2} e_{s}^{ \pm}+\frac{A_{c}}{2}\left(d_{2}+\beta d_{1}\right) e_{s}^{ \pm},
\end{aligned}
$$

$\mathrm{d}_{1}, \mathrm{~d}_{2}$ sont les inversions de populations adimensionnées associées aux ondes laser et Brillouin, $e_{a}^{ \pm}$est l'amplitude adimensionné du champ acoustique, $e_{p}^{ \pm}$et $e_{s}^{ \pm}$sont les amplitudes adimensionnés des champs optiques laser et Brillouin. Les signes + et désignent respectivement les ondes aller et retour. $t$ et $\mathrm{z}$ sont les variables adimensionnées temps et position respectivement. $p$ est le paramètre du pompage considéré uniforme le long de la cavité. $a_{1}=\frac{T_{r}}{\tau}, A_{c}=\sigma d_{0} L, g_{c}=\frac{g_{B} L\left|A_{0}\right|^{2}}{2}$ avec $g_{B}=\frac{2 g_{e} g_{a} c_{a}}{\Gamma}, \alpha_{c}=\alpha L, \alpha_{a}=\frac{T_{r} \Gamma}{2}, \mathrm{~T}_{\mathrm{r}}=48 \mathrm{~ns}$ est le temps de transit dans la cavité. $d_{0}=10^{24} \mathrm{~m}^{-3}$ est la densité des ions dopants, $\tau=800 \mu$ s est la durée de vie de l'inversion de population, $\mathrm{L}=10 \mathrm{~m}$ longueur de la cavité, $\sigma$ est la section efficace d'émission, $\gamma$ est le paramètre de dichroïsme du pompage. $\beta$ est le paramètre de saturation croisée. $\Gamma$ est le coefficient d'amortissement de l'onde sonore. $\alpha=0.045 \mathrm{~m}^{-1}$ est le coefficient d'absorption, $g_{e}$ et $g_{a}$ sont les coefficients de couplage optique et acoustique 
respectivement. $g_{c}$ est le gain Brillouin adimensionné. $g_{B}$ la constante du gain Brillouin dans la silice. Les conditions aux limites imposées par le dispositif expérimental s'écrivent:

$$
\begin{aligned}
& e_{p}^{+}(0, t)=\sqrt{R_{1}} e_{p}^{-}(0, t), \\
& e_{p}^{-}(l, t)=\sqrt{R_{2}} e_{p}^{+}(l, t), \\
& e_{s}^{+}(0, t)=\sqrt{R_{1}} e_{s}^{-}(0, t), \\
& e_{s}^{-}(l, t)=\sqrt{R_{2}} e_{s}^{+}(l, t) .
\end{aligned}
$$

$\mathrm{R}_{1}$ et $\mathrm{R}_{2}$ sont les coefficients réflexion des deux miroirs de la cavité optique.

Le seuil laser est déduit analytiquement du modèle (1):

$$
p_{t h}=\frac{a_{1}}{1+\beta \gamma}\left[1+\beta+\frac{1}{A_{c}}\left(\alpha_{c}+\frac{1}{2 \ell} \ln \frac{1}{\sqrt{R_{1}} \sqrt{R_{2}}}\right)\right]
$$

Le système d'équations est intégré numériquement en utilisant la méthode de Runge Kutta d'ordre quatre pour le temps et la méthode des différences finies à cinq points pour la position. On s'intéresse à l'évolution temporelle de l'intensité à la sortie de la cavité optique. Pour les simulations numériques on se place dans le cas pratique d'un laser à fibre double gaine dopée Ytterbium.

\section{RESULTATS NUMERIQUES}

Une dynamique très riche et variée, est obtenue en variant le taux de pompage, Pour les taux de pompage les plus élevés un régime quasi périodique s'installe pour une valeur de pompage $r=5,3$. Comme on peut le voir sur la figure (1), La construction du portrait de phase en trois dimensions est faite en utilisant les coordonnées décalées. Elle illustre un tore, voir figure (2). Le diagramme de premier retour montre une courbe fermée mais de faible " ellipticité », et le diagramme de vingtième retour présente une courbe fermé proche de la géométrie habituelle d'un régime quasi périodique, voir figure (3). Le caractère unidimensionnel de diagramme du vingtième retour est la signature du régime quasi périodique à deux fréquences comme le confirme le spectre de Fourier de l'amplitude de l'intensité, voir figure (4).

Pour un taux de pompage $r=5,7$ le portrait de phase présente un tore mais néanmoins particulier car il se replie sur lui-même, voir figure (5). Ce qui se voit sur le diagramme de premier retour et du vingtième. L'application montre une sorte de dédoublement qui montre que la trajectoire évolue sur les deux parties du tore «repliée », et un 
recoupement de la courbe dû à l'importance de l'harmonique d'ordre deux [2], voir figure (6) et figure (7). Le dédoublement illustré suggère que ce tore est plongé dans un espace de phase de dimension au moins égale à quatre au lieu de trois [3].

\section{CONCLUSION}

La prise en compte de la dynamique temporelle de l'onde acoustique dans l'étude de l'instabilité Brillouin dans un laser à fibre de puissance, nous a permet d'identifier un régime nouveau dans le fonctionnement dynamique du laser tel que le régime quasi périodique. Ce dernier provient de la présence de deux ou plusieurs fréquences incommensurables, la trajectoire évolue ainsi selon un tore dans l'espace des phases comme on l'a montré précédemment.

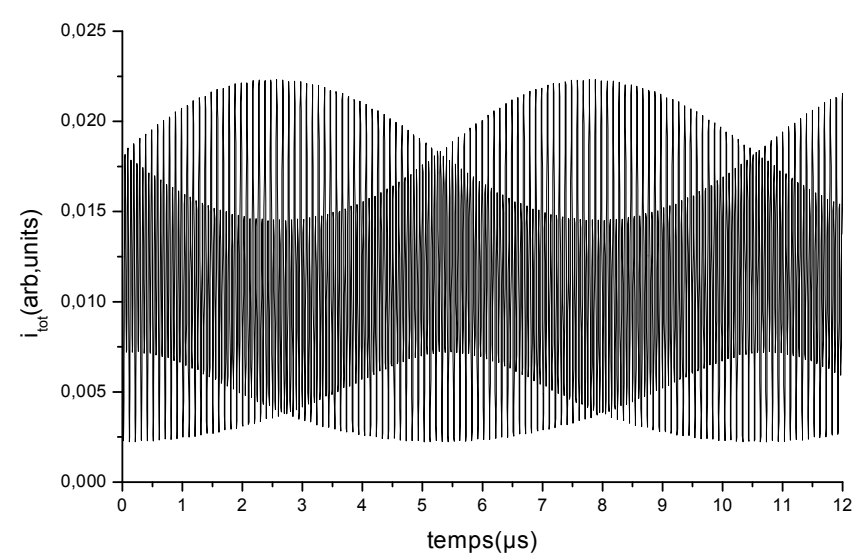

Fig.1. Evolution temporelle à $\mathrm{z}=\mathrm{L}$ de l'intensité totale pour un taux du pompage $r=5,3$

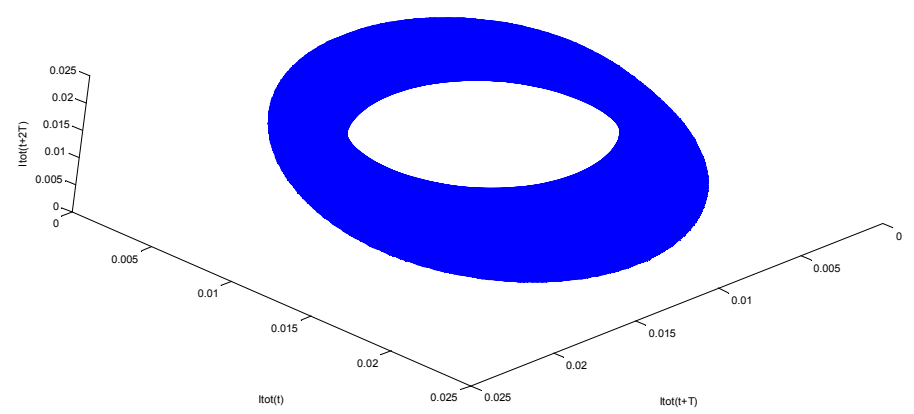

Fig.2. portrait de phase de l'intensité totale $\left(i^{+}+\mathrm{s}^{+}\right)$pour un taux de pompage $\mathrm{r}=5,3$. 


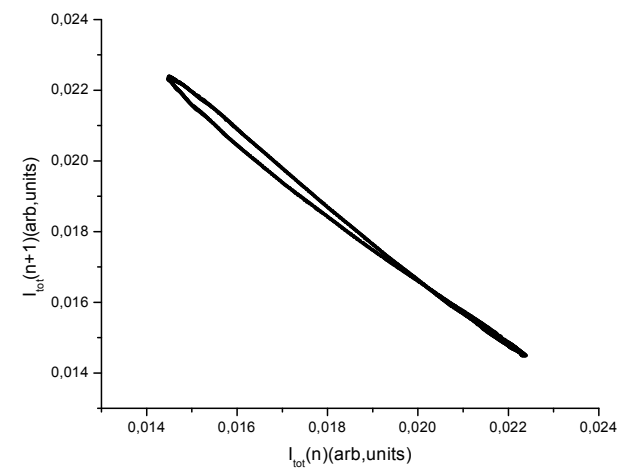

(a)

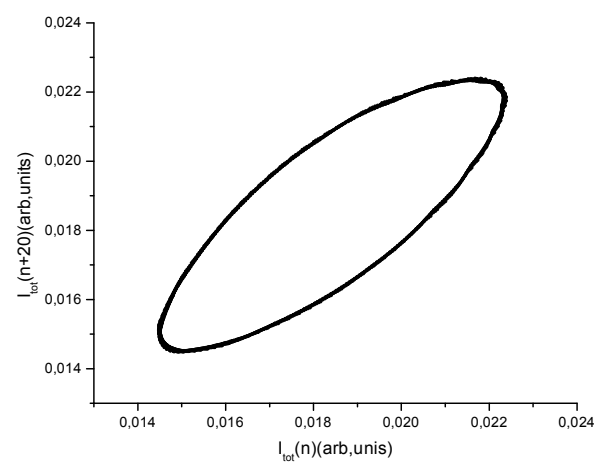

(b)

Fig.3. (a)Diagramme de premier retour, (b) diagramme de vingtième retour, pour un taux du pompage $r=5,3$.

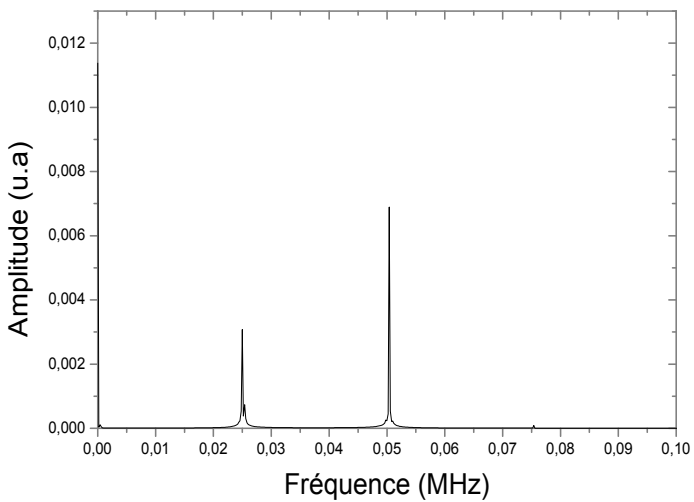

Fig.4. le spectre de l'amplitude de l'intensité pour un taux de pompage $r=5,3$.

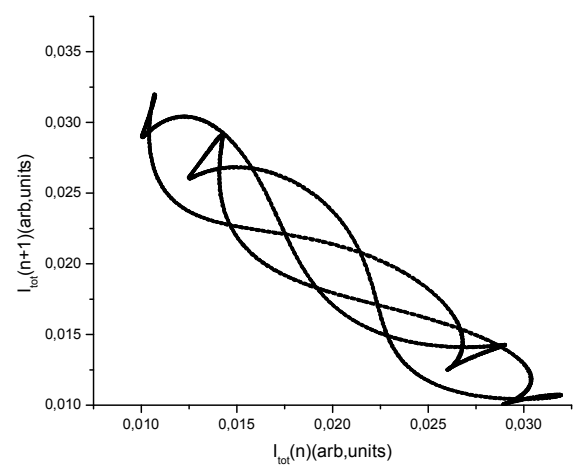

Fig.6. Diagramme du premier retour pour un taux de pompage $r=5,7$.

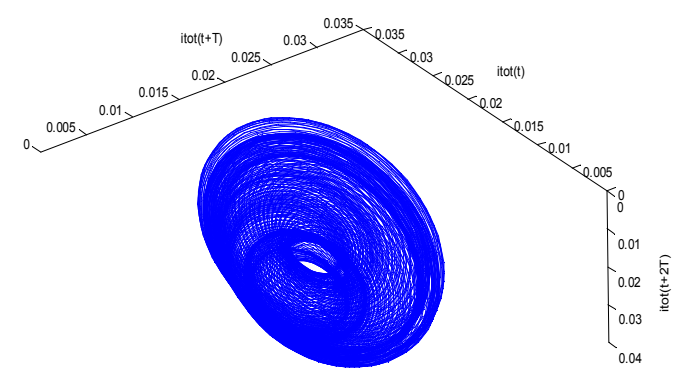

Fig.5. Portrait de phase del'intensité totale pour un taux du pompage $r=5,7$.

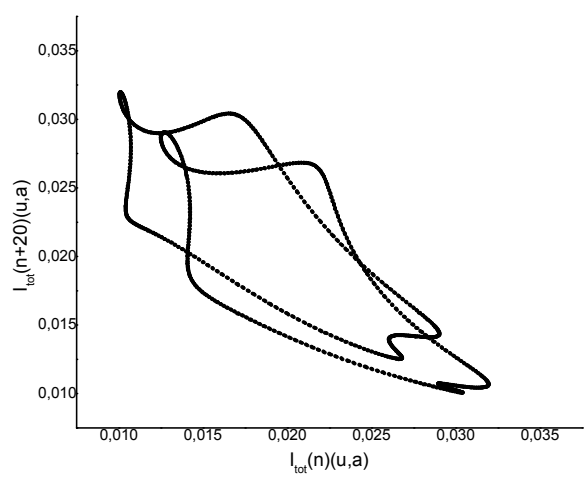

Fig.7. diagramme du vingtième retour pour un taux de pompage $r=5,7$. 


\section{RÉFÉRENCES}

[1] Mallek D., Kellou A., Leblond H., Chanchez F. Brillouin instabilities in continously pumped high power fiber lasers. Journal of Nonlinear Optical Physics \& Materials. 2009, 18(1), 111-120.

[2] Bergé P., Pomeau Y., Vidal Ch. 1992, L'ordre dans un chaos, Hermann éditeur des sciences et des arts.

[3] Arbanal H. D. I., Brown R., Sidorowich J. J., and Tsimring L. S. The analysis of observed chaotic data in physical systems. Reviews of Modern Physics. Octobre 1993, 65 . 


\section{L'INSTABILITE BRILLOUIN DANS LES LASERS A FIBRE DOPEE DE PUISSANCE}

\section{RESUME}

Avec l'émergence des fibres dopées aux terres rares, et spécialement la fibre double gaine, l'intérêt pour l'effet Brillouin a repris de l'importance. L'amplification d'un signal continu dans les fibres dopées aux terres rares génère des intensités élevées qui donnent lieu à la génération d'ondes contra-propagatives (l'onde Stokes). L'influence de ce processus est étudiée dans ce présent travail.

Mots-clés: Diffusion Brillouin stimulée, étude dynamique, laser à fibre de puissance.

How to cite this article

Mallek-Bouras D, Kellou A, Leblond H and Sanchez F. The Brillouin instability in fiber lasers doped by power. J Fundam Appl Sci. 2012, 4(1), 46-52. 